\title{
Improvement of the mechanical performance of Fergoug dam sediments treated for reuse in road engineering
}

\author{
LAROUCI Abdelkader ${ }^{1}$, SENHADJI Yassine ${ }^{1}$, LAOUFI Laïd ${ }^{1}$, BENAZZOUK Amar ${ }^{2}$ \\ ${ }^{1}$ Université de Mustapha Stambouli de MASCARA, Département de Génie Civil, Algérie \\ ${ }^{2}$ Université de Picardie jules Verne IUT d'AMIENS, Département de Génie Civil, France
}

\begin{abstract}
The phenomenon of siltation is one of the main problems facing many dams throughout the world, and particularly in Algeria. The mud, before and even after extraction, is always harmful. Indeed, mud is a harmful material, first for dams, because it reduces their capacity of water storage, and second, for the environment following the desilting operations that generate large quantities of materials that occupy and sometimes pollute large areas. However, these materials can be exploited differently and used in other fields. The envisaged research work relates to real cases of silted dams, such as the dam of Fergoug (western Algeria) whose siltation rate is very high; it is estimated at $97 \%$ of its initial capacity which, according to the National Agency for Dams (Agence Nationale des Barrages - ANB), is equal to 18 million $\mathrm{m}^{3}$. The large quantities of silt extracted present an environmental problem, and its use as a local raw material for the manufacture of civil engineering materials can contribute to solve this problem. The vulnerable lands of the sub-catchment of Wadi Fergoug (Fergoug River) extend over an area of $122 \mathrm{~km}^{2}$, from a total surface of $8340 \mathrm{~km}^{2}$ for the dam catchment area. There is a great diversity of superficial formations with predominantly clay soils from marly formations. The rate of specific erosion has increased to $160 \mathrm{~T} / \mathrm{km}^{2} /$ year due to irregular annual rainfall resulting from a succession of dry and wet years. Sedimentary materials, which are found in considerable quantities, were collected at the foot of the dam, on its right bank. This study attempts to find a recycling pathway for these sediments. The objective of the present work is to investigate the behavior of silt from the dam of Fergoug (Algeria) for the purpose of using it in road construction (foundation and base layers). The method adopted is to reconstitute, in the laboratory, samples of mixtures containing road aggregates with different proportions of silt. These samples are then subjected to various tests (Normal modified Proctor, Atterberg limits, VBS, Particle size analysis, Sedimentation, DRX etc ...). The results of the tests indicate that some of the prepared mixtures present very interesting properties. These results satisfy the current recommendations of the technical guides; they also corroborate their use in road construction. Keywords: Dredged sediments; Valorization; Road construction; California bearing ratio (CBR) index; Mechanical behavior; Environment.
\end{abstract}

\section{Introduction}

Le barrage de Fergoug est situé dans une zone montagneuse caractérisée par un climat agressif avec alternance de période très chaudes et froides, en plus des pluies dévastatrices pour les sols du bassin versant nus de toute couverture végétale. Ainsi les phénomènes d'érosion interne et externe sont très accrus dans la région et par conséquent, le barrage en question est pratiquement rempli de vase.

Pour y remédier à ce problème d'envasement, plusieurs recherches sont lancées dans ce domaine. Certains travaux sont réalisés avant l'envasement dans le but de proposer des solutions réduisant ce phénomène $[1,2]$.D'autres par contre, sont réalisés après envasements dans le but de la réutilisation de la vase dans les différentes activités, notamment dans le domaine du génie civil [3-6].

Cette étude a pour objectif la valorisation des sédiments de barrage dans le domaine routier en vue de leur utilisation dans les corps de chaussées (couches de fondation et couches de base) [7].

\section{Intérêts du travail réalisé}

Ce travail peut avoir plusieurs intérêts, tant sur le plan économique et environnemental, à savoir :

L'exploitation des quantités importantes de vase évacuées périodiquement par les vannes de fond de barrages dont les effets sont néfastes pour l'environnement. 
- Par conséquent, la récupération des espaces occupés par ces matériaux pour une éventuelle utilisation dans des autres activités.

- Le renforcement des secteurs du génie civil et des travaux publics par de nouvelles sources de granulats routiers, sachant que les sources habituelles alluvionnaires restent parfois incapables de satisfaire les besoins nationaux.

\section{Programme expérimental}

\subsection{Matériaux utilisés}

\subsubsection{La vase}

La vase est défini comme étant un ensemble d'élément déposes par l'eau, le vent, la glace qui proviennent de l'usure des continents, c'est-à-dire de la destruction de roches ou d'être vivants. La destruction se fait par mécanismes physique produisant la fragmentation des matériaux et des réactions chimiques donnant des solutions de lessivage (altération chimique).

Le terme «vase» englobe une large famille de sédiments représentée par trois phases; une phase liquide, colloïdale et minérale. Constituée de particules solides dont l'origine peut être naturelle ou anthropique.

\subsubsection{Caractéristiques physico-chimique}

Toute la vase prélevée dans la zone de rejet est convenablement préparée, elle est séchée $\left(60^{\circ} \mathrm{C}\right)$ et réduite en poudre, ensuite tamisée au tamis $1 \mathrm{~mm}$.

Les essais de caractérisation physico chimique sont réalisés au laboratoire LNHC (Laboratoire National de l'Habitat et de Construction) Unité de Sidi Bel Abbes. Elles sont résumées dans le tableau 1.

Tableau 1. Caractéristiques physico-chimique des sédiments du barrage Fergoug

\begin{tabular}{|c|c|c|}
\hline Caractéristiques & Designation & Résultats \\
\hline Teneur en eau naturelle & $(\%)$ & 52 \\
\hline Limite de liquidité & $\mathrm{W}_{\mathrm{L}}(\%)$ & 62,54 \\
\hline Limite de plasticité & $\mathrm{W}_{\mathrm{P}}(\%)$ & 29,29 \\
\hline $\begin{array}{c}\text { Indice de plasticité } \\
\mathrm{I}_{\mathrm{P}}(\%)\end{array}$ & 33,26 \\
\hline $\begin{array}{c}\text { Masse volumique } \\
\text { sèche }\end{array}$ & $\rho_{\mathrm{OPM}}\left(\mathrm{g} / \mathrm{cm}^{3}\right)$ & 1,779 \\
\hline $\begin{array}{c}\text { Teneur en eau de } \\
\text { l'optimum Proctor }\end{array}$ & $\mathrm{W}_{\mathrm{OPM}}(\%)$ & 19,30 \\
\hline $\begin{array}{c}\text { Valeur du bleu } \\
\text { méthylène }\end{array}$ & \begin{tabular}{c}
$\left.\mathrm{VBS}_{(\mathrm{g} / 100 \mathrm{~g}} \mathrm{dul}\right)$ \\
\hline $\begin{array}{c}\text { Carbonates } \\
\mathrm{CaCO}_{3}(\%)\end{array}$
\end{tabular} & 2,58 \\
\hline $\begin{array}{c}\text { Matière Organique } \\
(\%)\end{array}$ & 3,15 \\
\hline
\end{tabular}

\subsubsection{Caractéristiques minéralogique}

Cette analyse nous permet d'identifier les minéraux présents dans notre vase, elle a été réalisée au laboratoire de la cimenterie 'Lafarge' d'Oggaz-Algérie.

L'analyse DRX de la vase brute nous montre l'existence de raies correspondants aux minéraux tels que le quartz $\left(\mathrm{SiO}_{2}\right)$, les carbonates types calcite $\left(\mathrm{CaCO}_{3}\right)$, et les argiles type Kaolinite $\left(\mathrm{Al}_{2} \mathrm{O}_{3} .2 \mathrm{SiO}_{2} .2 \mathrm{H}_{2} \mathrm{O}\right)$ et l'illite, ainsi que la Dolomite $\mathrm{CaMg}\left(\mathrm{CO}_{3}\right)_{2}$ (Figure 1).

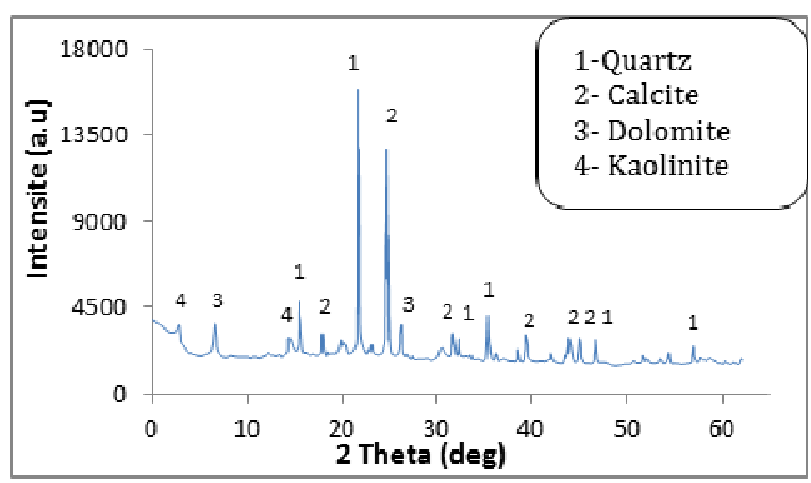

Fig. 1. Analyse minéralogique de la vase par DRX.

\subsubsection{Les granulats routiers}

Les granulats routiers utilisés sont prélevé dans le tronçon de la pénétrante autoroutière qui relier la ville de Mascara a l'Autoroute Est-Ouest publics ce qui est en cours de réalisation. C'est les granulats utilisés pour la construction routière dans la région Mascara et Oran (Algérie). Cette dernière est caractérisée par la courbe granulométrique présentée sur la Fig. 2. Selon la classification des matériaux utilisables dans la construction des remblais et des couches de forme d'infrastructures routières (NF P 11-300), les granulats routiers utilisés se présentent sous forme d'une grave propre mal graduée, de granulométrie uniforme.

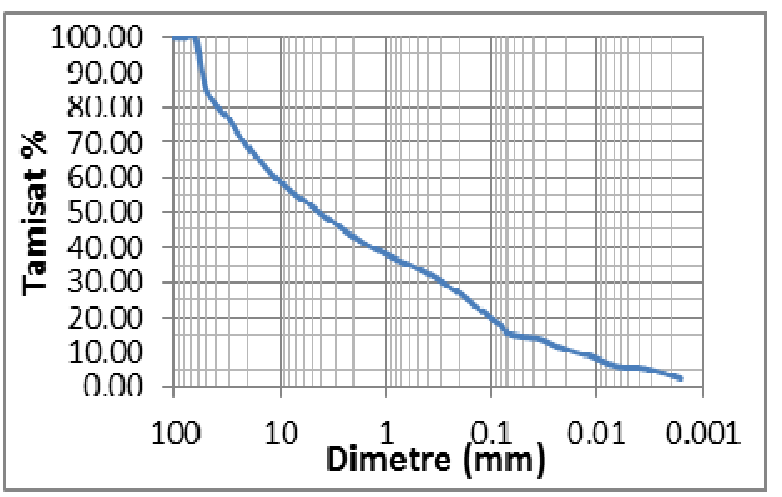

Fig. 2. Courbe granulométrique du matériau utilisé.

\section{Echantillons préparés et essais réalisés}

Les échantillons soumis aux essais mécaniques sont préparés à base de granulats routiers et de sédiments de barrage, sous forme de mélanges selon les proportions résumé dans le tableau suivants : 
Tableau 2. Les mélanges préparés avec le granulat routier et la vase du barrage.

\begin{tabular}{|l|c|c|}
\hline & Granulats routiers (\%) & Vase (\%) \\
\hline Echantillon 1 & 100 & 0 \\
\hline Echantillon 2 & 95 & 5 \\
\hline Echantillon 3 & 90 & 10 \\
\hline Echantillon 4 & 85 & 15 \\
\hline Echantillon 5 & 80 & 20 \\
\hline Echantillon 6 & 75 & 25 \\
\hline
\end{tabular}

Afin d'étudier le comportement des mélanges sous les principales sollicitations routières en fonction de leur teneur en sédiments, les échantillons préparés sont soumis aux essais physiques et mécaniques sont déterminés conformément aux normes françaises.

- Essais de limite d'Atterberg (NF P94-051);

- Essais de compactage au Proctor Normal et Proctor modifié (NF P94-093) ;

- Essais de portance immédiat (IPI) et essais de portance après immersion $\left(\mathrm{CBR}_{\mathrm{imm}}\right)$ (NF P94-078).

\section{Résultats et analyses}

\subsection{Effet de la vase sur les limites d'Atterberg}

Les résultats des essais sur les limites d'Atterberg aux différents pourcentages sont montrés dans le tableau 3 et sur la fig. 3. A travers l'allure de la courbe dans la fig. 3, on peut clairement apprécier la tendance progressive de l'indice de plasticité des différents échantillons, on en déduit que l'augmentation du pourcentage de la vase de barrage à un effet direct sur l'indice de plasticité du matériau composé Granulat routier-Vase.

D'après les résultats trouvés, On montre un changement remarquable de l'indice de plasticité après l'incorporation du vase. Il est évident qu'une addition de $15 \%$ et $25 \%$ du vase pour une augmentation de $51,84 \%$ et $97.07 \%$ respectivement. L'augmentation de l'indice de plasticité dans notre échantillon est indiqué une amélioration dans la plasticité du sol. L'indice de plasticité est très fortement lié à la quantité et à la nature des minéraux argileux présents dans le sol et à la surface spécifique des sols.

Tableau 3 : Valeurs des limites d'Atterberg

\begin{tabular}{|c|c|c|c|}
\hline L'échantillon & $\mathbf{W}_{\mathbf{L}}$ & $\mathbf{W p}$ & $\mathbf{I p}$ \\
\hline $\mathbf{1}$ & 18.62 & 13.47 & 5.15 \\
\hline $\mathbf{2}$ & 19.41 & 13.64 & 5.77 \\
\hline $\mathbf{3}$ & 20.42 & 13.83 & 6,59 \\
\hline $\mathbf{4}$ & 21.84 & 14.02 & 7.82 \\
\hline $\mathbf{5}$ & 22.81 & 14.15 & 8.66 \\
\hline $\mathbf{6}$ & 24.51 & 14.46 & 10,13 \\
\hline
\end{tabular}

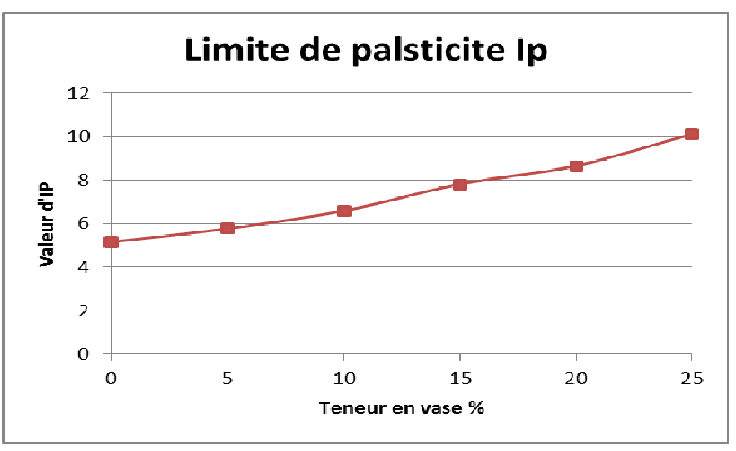

Fig. 3. L'indice de plasticité (Ip) de la vase de barrage de Fergoug

\subsection{Essais de compactage au Proctor Normal et Proctor Modifié}

Les essais Proctor sont réalisés selon la norme (NF $\mathrm{P}$ 94-093). Ils permettent de montrer l'aptitude au compactage des mélanges étudiés en fonction de leurs teneurs en vase. Les courbes de compactage (normal et modifié) correspondant aux différents mélanges sont présentées sur les figs. 4 et 5 (Proctor normal) est les figs. 6 et 7 (Proctor modifié).

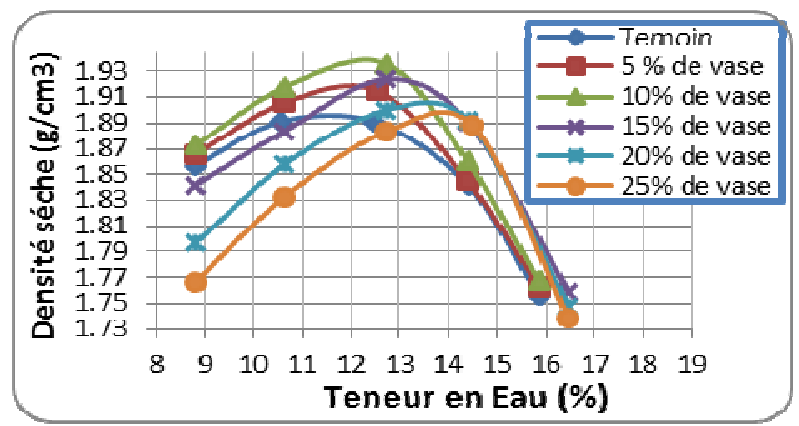

Fig. 4. Courbes de compactage au Proctor normal.

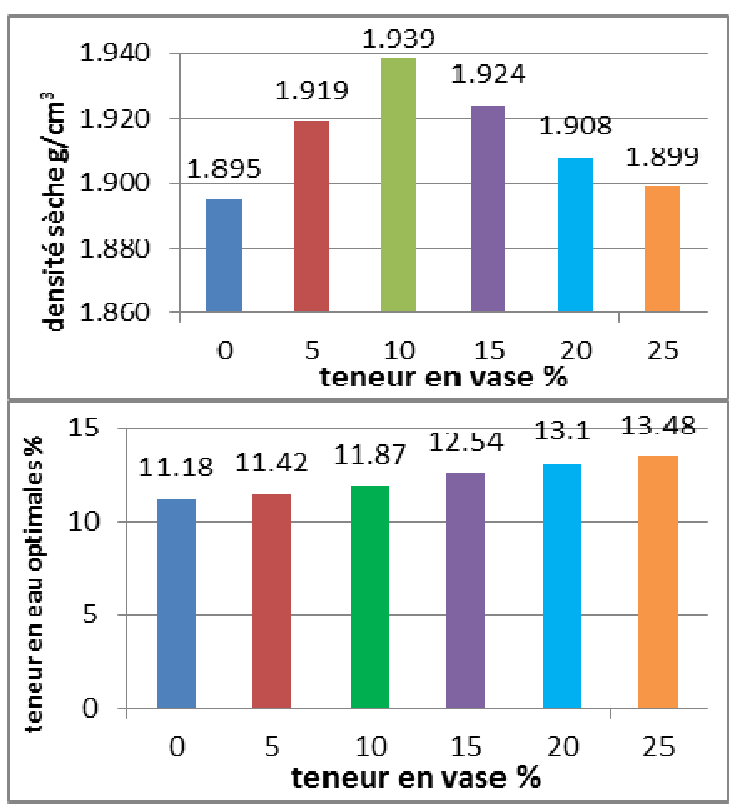

Fig. 5. Caractéristiques de compactage en fonction de la teneur en sédiments (Proctor normal). 
A première vue, nous pouvons constater que les courbes Proctor représentant les différents mélanges se distinguent clairement de celle correspondant aux granulats routiers seuls. Par rapport à cette dernière, nous pouvons remarquer que les courbes représentant les mélanges de $5,10,15,20$ et $25 \%$ de sédiments sont situées pratiquement du coté humide.

L'évolution de la densité sèche maximale des mélanges étudiés en fonction de leurs teneurs en sédiments est présentée avec plus de précision sur Figs. 4 et 5 (Proctor normal) et Figs. 6 et 7 (Proctor modifié).

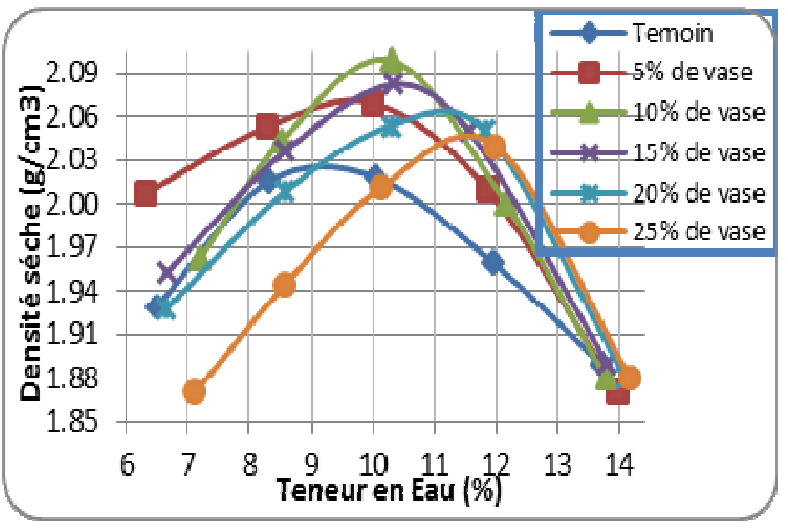

Fig. 6. Courbes de compactage au Proctor modifier.

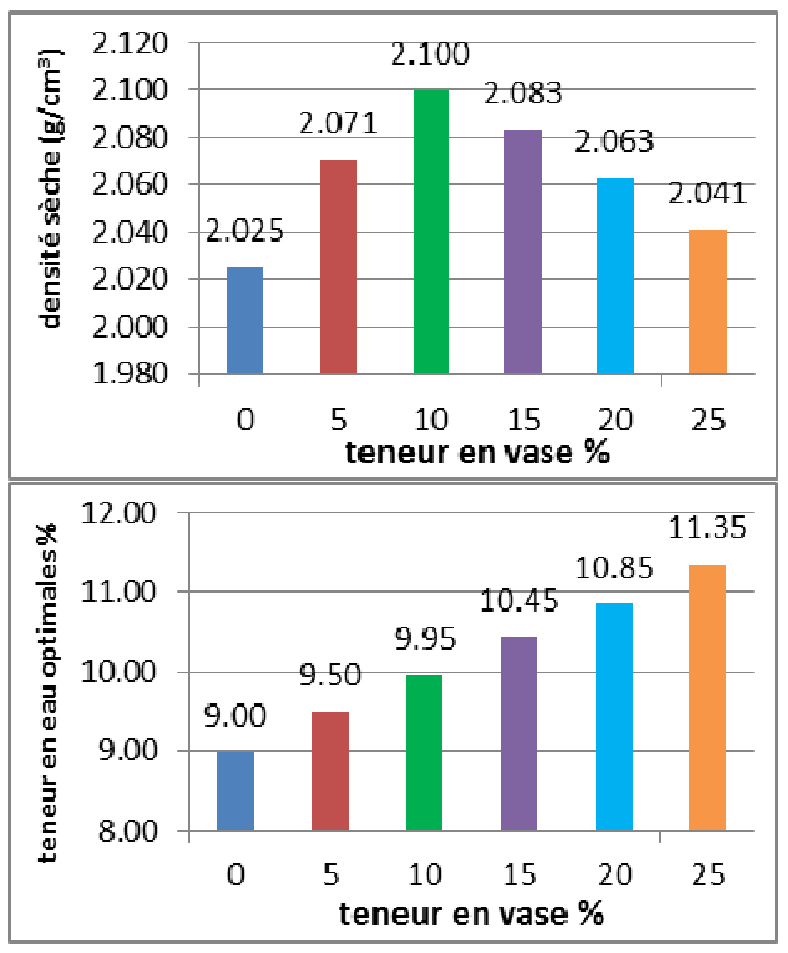

Fig. 7. Caractéristiques de compactage en fonction de la teneur en sédiments (Proctor modifié).

La densité sèche des mélanges s'améliore de plus en plus jusqu'à atteindre une valeur maximale pour une teneur en sédiments de $10 \%$. Au-delà de cette proportion, les mélanges commencent à perdre de leurs densités, toutefois, ces densités restent supérieures aux témoins et pour Proctor modifier reste supérieur a 2, de tels résultats sont assez suffisants pour un matériau routier [8]
Ceci est dû au fait que cette proportion de vase adéquate rentre facilement dans les vides entre les granulats routiers ce qui réduit leur volume (vides) et rend les mélanges plus denses.

L'augmentation de la teneur en eau optimale des mélanges est due à l'accroissement de la surface totale des particules des mélanges par les grains fins de la vase additionnée.

Une proportion supérieure à $10 \%$ de sédiments, dépasse le volume des vides existants entres les granulats routiers, la densité sèche elle commence a réduit de plus en plus en fonction de l'ajout de la vase, la raison la diminution de la densité sèche maximum est le faible poids volumique de la vase $\left(1.779 \mathrm{t} / \mathrm{m}^{3}\right)$ par rapport les granulats routière seul $\left(2.025 \mathrm{t} / \mathrm{m}^{3}\right)$.

On remarque que l'augmentation de la teneur en sédiments dans les granulats, la courbe de compactage soit à l'OPM ou l'OPN présente de plus en plus une forme accentuée, ce qui explique la sensibilité du mélange à l'eau par rapport à l'état naturel [9].

\subsection{Essais de portance immédiats (IPI)}

Les courbes représentant les forces en fonction des enfoncements pour les mélanges dont la teneur en sédiments de 5 à $10 \%$ se distingue clairement sur la Fig. 8 pour (Proctor normal) fig. 9 (Proctor modifié).

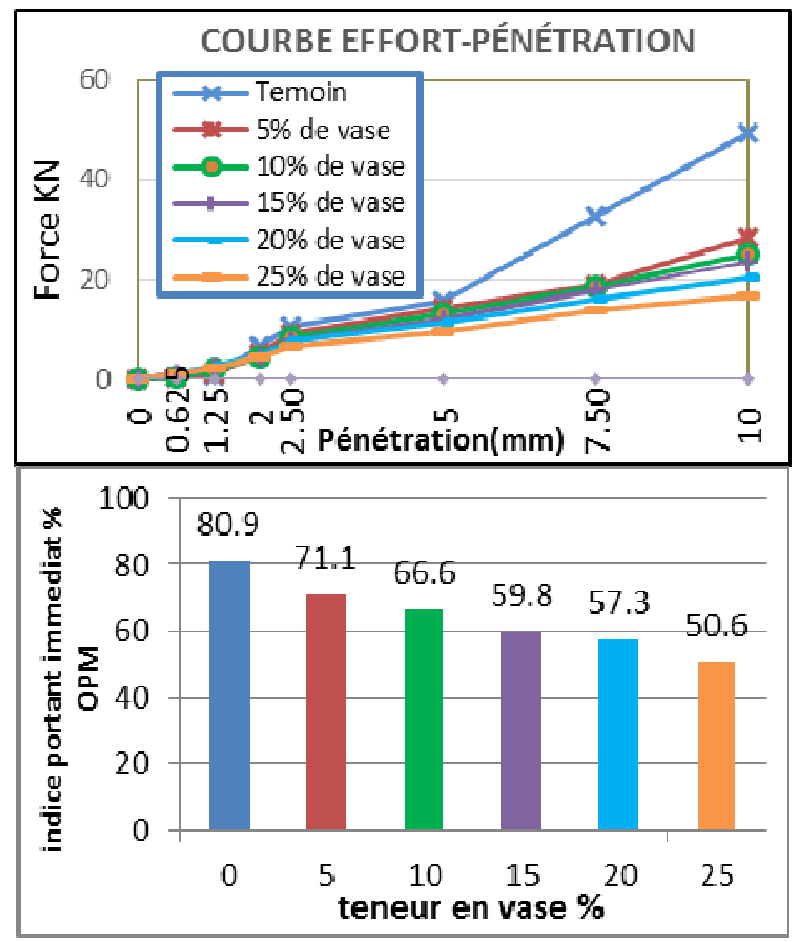

Fig. 8. Effort de pénétration IPI (OPM)

La portance des mélanges empirés dès le premier ajout de sédiments. Néanmoins, les mélanges à $5 \%$ de sédiments présentent les portances les plus élevées que les autres mélange $(10,15,20$, et $25 \%)$, malgré cela il reste toujours inferieur par rapport des granulats routiers seuls sachant que ces derniers sont constatés les plus denses au cours des essais de compactage. 


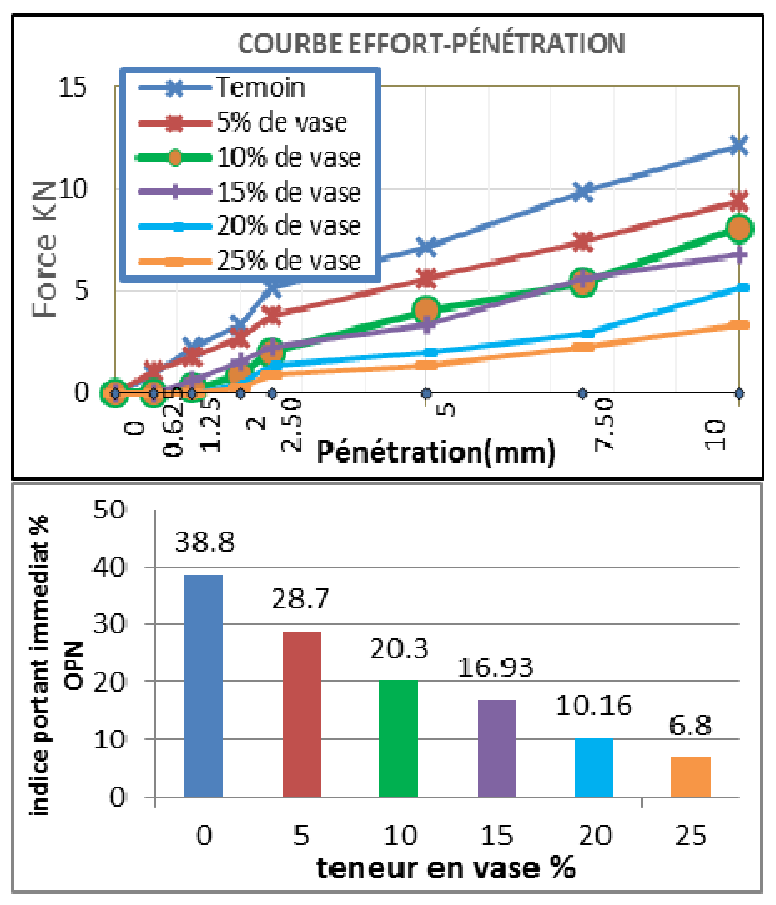

Fig. 9. Effort de pénétration IPI (OPN)

La raison de la diminution de la portance du sol c'est la vase qui entoure les granulats routiers, elle est facile le poinçonnement de plus en plus en fonction de l'ajout des sédiments.

\subsection{Essais De Portance CBR Après Immersion}

Les essais CBR après immersion réalisés selon la norme NF P 94-078, permettent d'évaluer la portance des mélanges sous la circulation des engins dans les plus mauvaises conditions hygrométriques (Indice CBR après immersion).

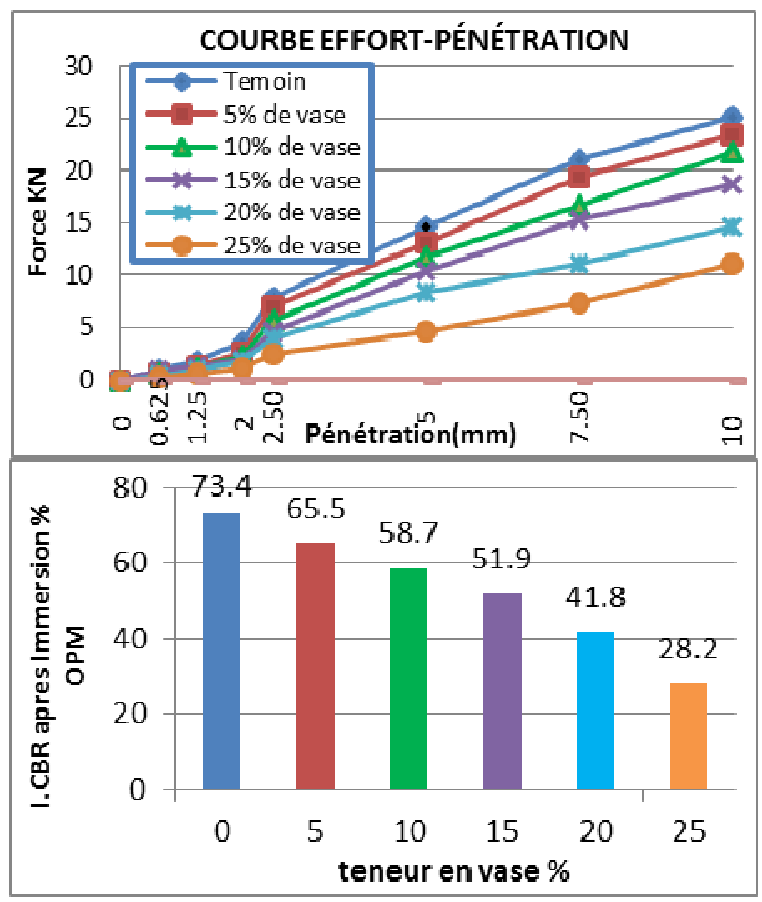

Fig. 10. Effort de pénétration CBR après Immersion (OPM)

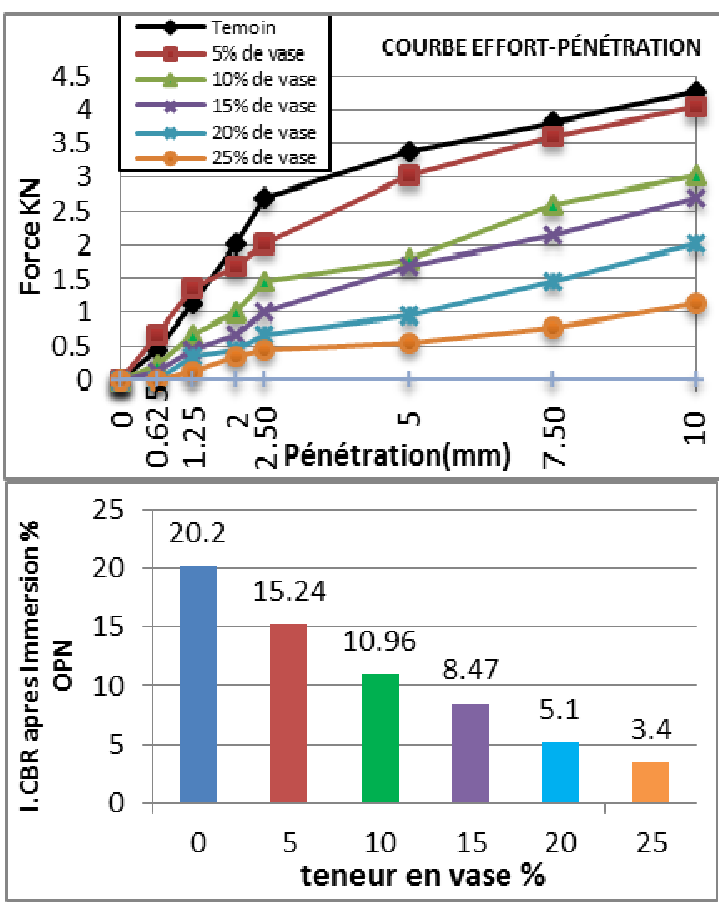

Fig. 11. Effort de pénétration CBR après Immersion (OPN)

La portance des mélanges diminue dès le premier ajout de vase. En effet, les courbes forces enfoncements correspondant aux mélanges (fig. 11 pour Proctor normal et fig. 10 pour Proctor modifié) s'écartent de plus en plus de celle correspondant aux granulats routiers seuls, notamment pour des teneurs en sédiments supérieures à $10 \%$ pour le Proctor normal et à $20 \%$ pour le Proctor modifier. La vase à l'état humide (immersion) même en faible proportion peut nuire à la portance des mélanges.

En effet, la vase humide présente une très faible résistance au poinçonnement. Les grains entourés de vase évitent facilement les pressions engendrées par le vérin de l'appareillage de poinçonnement en se déplaçant vers les points de faibles pressions, ces déplacements sont de plus en plus favorisés avec l'ajout de vase.

En ce qui concerne le gonflement relatif $(G)$ en présence d'eau ; on peut dire qu'il est négligeable dans tous les cas. En effet le gonflement maximal est enregistrée dans le cas du mélange (granulats routiers + vase), néanmoins, il ne dépasse pas les $0,3 \%$ pour le Proctor normal et $0.27 \%$ pour le Proctor modifier.

\section{Conclusion}

Les travaux de recherche présentés dans cet article entrent dans le cadre général de la valorisation sédiments dragué des barrages en Algérie. Notre contribution est essentiellement centrée sur le cas de barrage Fergoug de la wilaya de Mascara ; Cette étude consiste à traiter le comportement des mélanges (granulats routiers + sédiments du barrage de Fergoug) sous sollicitation routière.

L'envasement des retenues de barrages en Algérie a pris une ampleur importante, limitant ainsi leur capacité (diminution importante du volume d'eau stockée). Le dé envasement est un moyen de lutte contre l'envasement 
néanmoins les conséquences engendrées sont le dépôt sédimentaire et les problèmes liés à son stockage.

L'objectif de valoriser de nouvelles sources de granulats d'une part et de préserver l'environnement d'autre part. Les résultats obtenus ont montré que les mélanges constitués de (granulats routiers +5 à $10 \%$ de sédiments) présentent un comportement très satisfaisant sous les différents types de sollicitations appliquées.

Ils peuvent par conséquent, être proposés dans la construction routière, en couches de fondations et en couches de base dans le cas des chaussées de faible à moyen trafic. Ce travail de recherche peut présenter un intérêt capital en géotechnique routière en Algérie, tant sur l'aspect économique et sur l'aspect écologique et environnemental.

\section{Références}

1. B. Abdellaoui, A. Merzouk, M. Aberkan, J. Albergel, Revue des sciences de l'eau 15 (2002), 737-748

2. W. Remini, B. Remini, Larhyss Journal 02 (2003) 45-54

3. N. Belas, O. Belaribi, A. Mebrouki, N. Bouhamou, Valorisation des sédiments de dragage dans les bétons, Séminaire International, innovation \& valorisation en génie civil \& matériaux de construction. INVACO2. Rabat, Maroc, (2011), pp. 23-25
4. A. SEMCHA, Valorisation des sédiments de dragage, Applications dans le BTP, cas du barrage de Fergoug, Thèse, Université de Reims Champagne- Ardenne, (2006).

5. F. Kazi Aoual, M. Ameur, B. Mekerta, A. Semcha, (2014). Caractérisation des sédiments de dragage du barrage de Bouhanifia pour une réutilisation, XIIIèmes Journées Nationales Génie Côtier - Génie Civil, Gestion et valorisation des sédiments marins, Dunkerque, France. pp. 999-1006.

6. Z. Labiod, B. Remini, M. Belaredj. Larhyss Journal, nº 03, Juin 2004, pp 7-12.

7. M. A. Bourabah, Comportement mécanique des sols fins, application à la valorisation des sédiments de barrages en technique routière, Thèse de doctorat en sciences, Université de Tlemcen, Algérie, (2012).

8. P. Autret. (1983). Institut des sciences et des techniques de l'équipement et de l'environnement pour le développement (ISTED), Laboratoire central des ponts et chaussées (LCPC), pp. 40.

9. A. Larouci, Y. Senhadji, L. Laoufi \& A. Benazzouk, Valorisation de la vase du barrage de Fergoug pour une construction routière. $3^{\text {rd }}$ International Symposium on Materials and Sustainable Development (ISMSD2017), 7 \& 8 Novembre 2017 - Boumerdes, Algérie. 